Annales Geophysicae (2002) 20: 609-618 (c) European Geophysical Society 2002

\title{
Evidence for the interplanetary electric potential? WIND observations of electrostatic fluctuations
}

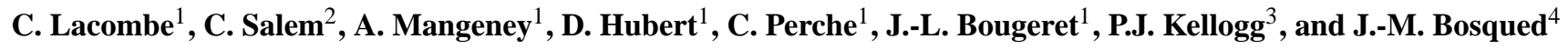 \\ ${ }^{1}$ DESPA/CNRS, Observatoire de Paris, 92195 Meudon, France \\ ${ }^{2}$ Space Science Laboratory, University of California, Berkeley, CA 94720, USA \\ ${ }^{3}$ School of Physics and Astronomy, University of Minnesota, Minneapolis, MN 55454, USA \\ ${ }^{4}$ CESR/CNRS, Université Paul Sabatier, BP 4346, 31029 Toulouse, France
}

Received: 14 June 2001 - Revised: 7 November 2001 - Accepted: 10 December 2001

\begin{abstract}
In the solar wind at $1 \mathrm{AU}$, coherent electrostatic waveforms in the ion acoustic frequency range $(\simeq 1 \mathrm{kHz})$ have been observed by the Time Domain Sampler (TDS) instrument on the Wind spacecraft. Small drops of electrostatic potential $\left(\Delta \Phi \geq 10^{-3} \mathrm{~V}\right)$ have been found across some of these waveforms, which can thus be considered as weak double layers (Mangeney et al., 1999). The rate of occurrence of these potential drops, at $1 \mathrm{AU}$, is estimated by a comparison of the TDS data with simultaneous data of another Wind instrument, the Thermal Noise Receiver (TNR), which measures continuously the thermal and non-thermal electric spectra above $4 \mathrm{kHz}$. We assume that the potential drops have a constant amplitude and a constant rate of occurrence between the Sun and the Earth. The total potential drop between the Sun and the Earth, which results from a succession of small potential drops during the Sun-Earth travel time, is then found to be about $300 \mathrm{~V}$ to $1000 \mathrm{~V}$, of the same order of magnitude as the interplanetary potential implied by a two-fluid or an exospheric model of the solar wind: the interplanetary potential may manifest itself as a succession of weak double layers. We also find that the hourly average of the energy of the non-thermal ion acoustic waves, observed on TNR between 4 and $6 \mathrm{kHz}$, is correlated to the interplanetary electrostatic field, parallel to the spiral magnetic field, calculated with a two-fluid model: this is another evidence of a relation between the interplanetary electrostatic field and the electrostatic fluctuations in the ion acoustic range. We have yet to discuss the role of the Doppler effect, which is strong for ion acoustic waves in the solar wind, and which can bias the measure of the ion acoustic wave energy in the narrow band $4-6 \mathrm{kHz}$.
\end{abstract}

Key words. Interplanetary physics (plasma waves and tur-

Correspondence to: C. Lacombe

(clacombe@despace.obspm.fr) bulence; solar wind plasma) Space plasma physics (electrostatic structures)

\section{Introduction}

The solar wind is a weakly collisional plasma so that the validity of the fluid equations that are generally used to describe its flow is questionable. It is usually argued that wave-particle interactions replace binary collisions and restore the fluid character of the flow by regulating the energy transport and dissipation (see Kellogg, 2000; Salem, 2000). Among the waves that can play a role in this respect, electrostatic waves with frequencies $f$ between the ion and electron plasma frequencies have been observed by several spacecraft in the free solar wind (i.e. in regions that are not magnetically connected to the Earth's bow shock). This broad band ion acoustic activity is an intermittent but almost permanent feature of the solar wind (Gurnett, 1991; Mangeney et al., 1999). Gurnett et al. (1979) argued that the intensity of this wave activity increases when the electron to proton temperature ratio $T_{e} / T_{p}$ increases, and when the electron heat flux increases, lending some support to the idea that these waves play a role in regulating, at least partially, the electron energy transport (see also Lin et al., 2001).

Exospheric models of the solar wind expansion assume, on the contrary, that the solar wind is essentially collisionless, at least above a certain height, and that a small amount of collisions or wave-particle interactions only helps to smooth the strong gradients that are eventually produced. In these models the fast moving electrons tend to escape from the corona, and an interplanetary electrostatic potential difference $\Phi_{S E}$ sets in between the solar corona and "infinity". The corresponding electric field is directed outward; it decelerates the outward propagating electrons and accelerates 
the protons. Thus, the presence of a potential difference between the corona and the Earth, $\Phi_{E X} \simeq 400 \mathrm{~V}$ or $500 \mathrm{~V}$ (Lemaire and Scherer, 1971; Pierrard et al., 2001), is a natural outcome of collisionless exospheric models of the solar wind. Note that there is also a potential difference in twofluid solar wind models, which is required to keep the electron fluid moving with the protons and is determined by the gradient of the electron pressure. The corresponding potential is $\Phi_{P G} \simeq 400 \mathrm{~V}$ to $600 \mathrm{~V}$, of the same order of magnitude as $\Phi_{E X}$. Such large-scale potentials cannot be measured directly in situ.

Recently, in the ion acoustic frequency range (0.2 to $10 \mathrm{kHz}$ ), the Time Domain Sampler (TDS) instrument on Wind has recorded electrostatic waveforms with a high temporal resolution. A first result of the TDS observations is that these waves are coherent and that the wave trains have scales of the order of a few tens of Debye lengths (Mangeney et al., 1999). Thus, the assumption that the waves have a random phase, usually made in the calculations of the wave-particle interactions, is not necessarily valid. A second result of the TDS observations is that a small jump of electrostatic potential $\Delta \Phi \geq 10^{-3} \mathrm{~V}$ is found across some of the coherent electrostatic waves (CEW). Such potential jumps imply that the corresponding CEW are weak double layers, with a charge separation on a scale comparable to the Debye length. The observed potentials usually drop towards Earth: they vary in the same sense as the interplanetary electrostatic potential $\Phi_{S E}$.

It is tempting to speculate that $\Phi_{S E}$ is actually the result of a succession of small potential drops in weak double layers (hereafter WDL), due to small charge separations between the protons and the escaping electrons (Salem et al., 1999). To check this hypothesis, we have to estimate the rate of occurrence of the WDL in the solar wind. This estimate cannot be made with the TDS instrument alone because the telemetry rate allows for the transmission of only one waveform every $10 \mathrm{~min}$. There is on Wind another wave instrument, the Thermal Noise Receiver (TNR), which measures continuously the spectra of electrostatic waves: at the ion acoustic frequencies, a spectrum is recorded every $4.5 \mathrm{~s}$. The TNR instrument is sensitive enough to measure both the non-thermal fluctuations corresponding to the coherent electrostatic waves seen on TDS, and the thermal fluctuations. Thus, a comparison of the spectral energy measured on TDS and of the average non-thermal spectral energy on TNR will give the rate of occurrence of WDL in the solar wind. We find that the resultant total potential drop is of the same order of magnitude $(300 \mathrm{~V}$ to $1000 \mathrm{~V})$ as the interplanetary potential $\Phi_{P G}$ or $\Phi_{E X}$.

Finally, to check whether the waves play a part in the solar wind energy transport, we study how the hourly average of the ion acoustic wave energy measured on TNR depends on the solar wind properties and on the electron heat flux. We do not find any correlation between the electrostatic energy $E^{2}$ between 4 and $6 \mathrm{kHz}$ and the electron heat flux or the normalised heat flux: if the heat flux is controlled by wave-particle interactions, then the interacting waves are probably not the ion acoustic waves (Lin et al., 1998; Gary et al., 1999). Similarly, we find no correlation between $E^{2}$ and $T_{e} / T_{p}$, a parameter that controls the damping of the ion acoustic waves in the linear Maxwell-Vlasov theory. On the other hand, a thorough analysis of the data has shown that there are three quantities that tend to increase when $E^{2}$ increases: the electron temperature $T_{e}$, the temperature anisotropy $T_{e \|}-T_{e \perp}$, and $\cos \chi$, where $\chi$ is the acute angle between the magnetic field and the GSE- $X$ direction (which is close to the solar wind direction). A possible interpretation can be found in the fact that the analytical expression of the two-fluid electrostatic potential $\Phi_{P G}$ in a spiral magnetic field itself depends on $T_{e}, T_{e \|}-T_{e \perp}$ and $\cos \chi$ (Pilipp et al., 1990). Therefore, we suggest that the energy $E^{2}$ of the electrostatic fluctuations in the ion acoustic range is related to the electric field $E_{I P}=-\nabla \Phi_{P G}$; besides, the electron Coulomb collisions may play a part in the generation of the ion acoustic waves since $E^{2}$ is larger when the collision frequency is weaker. The (unknown) instability generating the ion acoustic waves and weak double layers could depend on the component $E_{I P \|}$ of this interplanetary electrostatic field parallel to the magnetic field, as well as on the collision rate.

Our suggestions about the influence of the collisions and of the interplanetary electrostatic potential on the energy of the ion acoustic waves have to be discussed. The Doppler effect, which depends on $\cos \chi$ and on the Debye length $\lambda_{D}$, can also play a part in the energy $E^{2}$ of the waves observed on TNR. The continuous measurement of these waves has only been made in a relatively narrow frequency band, 4 to $6 \mathrm{kHz}$, above the most probable frequency of the ion acoustic waves, which is around $1 \mathrm{kHz}$ on TDS. An increase in the Doppler shift on $1 \mathrm{kHz}$ waves will give an apparent increase in $E^{2}$, even if there is no increase in the wave intensity in the whole ion acoustic range.

\section{The data}

The WAVES experiment on the Wind spacecraft measures the electric and magnetic plasma waves in a large range of frequencies (Bougeret et al., 1995). In the present study, we consider the electric field measurements provided by two WAVES instruments (TDS and TNR) with the $x$ antenna, a wire dipole of physical length $2 L_{x}$ tip-to-tip, spinning in the ecliptic plane $\left(L_{x}=50 \mathrm{~m}\right)$.

The TDS instrument detects all the electric waveforms above a programmable threshold, but only a few waveforms are transmitted to the ground. We consider high bit rate data, for which the 2048 points of the waveform are sampled during $17 \mathrm{~ms}$. During the considered interval in 1995, the transmitted event is not the most intense but the most recently recorded, every $10 \mathrm{~min}$. To obtain the electric field $E$ along the $x$ antenna, we divide the measured potential difference $V$ at the antenna terminals by the length $L_{x}$. We calculate the average spectrum of more than 2000 waveforms; $29 \%$ of these waveforms are weak double layers, with a potential drop $\geq 10^{-3} \mathrm{~V}$. 

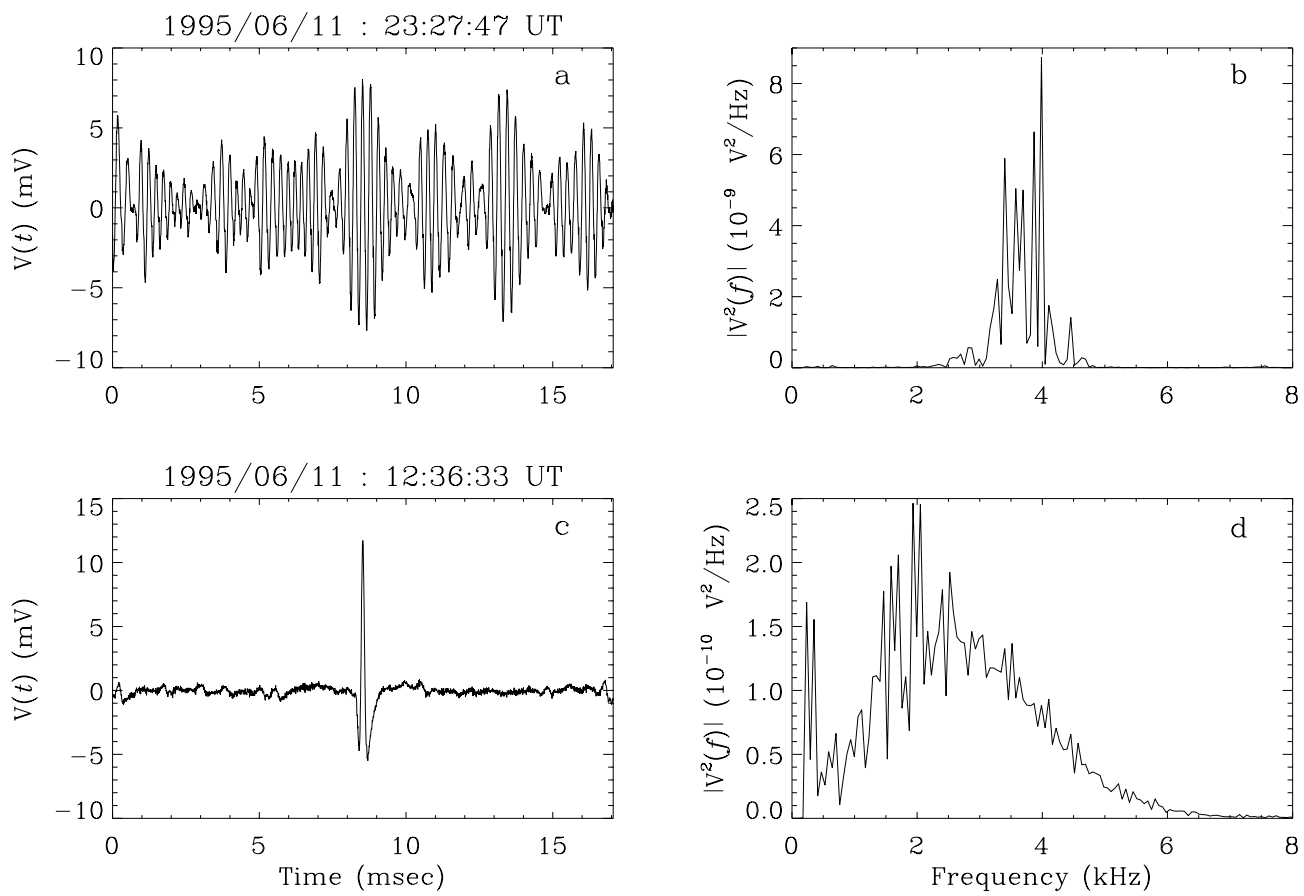

Fig. 1. Electrostatic waveforms observed by the TDS instrument in the solar wind. (a) and (b) packets of quasi-sinusoidal waves, and their spectrum. (c) and (d) an isolated weak double layer and its spectrum.

In its lowest frequency band ( 32 channels between 4 and $16 \mathrm{kHz}$ ) the TNR receiver measures a spectrum in $\mathrm{V}^{2} / \mathrm{Hz}$ every $4.5 \mathrm{~s}$ with an integration time of $1.472 \mathrm{~s}$. With the TNR data, the square electric field $E^{2}\left(\mathrm{~V}^{2} \mathrm{~m}^{-2} \mathrm{~Hz}^{-1}\right)$ is usually obtained by dividing $V^{2}\left(\mathrm{~V}^{2} / \mathrm{Hz}\right)$ by $L_{x}^{2}$. This leads to an underestimation of $E^{2}$ for small wavelengths $\lambda$. The response of a dipole antenna is a function of $k L_{x}$, where $k=2 \pi / \lambda$ is the wave vector (see Fig. B1, Scudder et al., 1986). When $k L_{x} \leq 1$, the potential is indeed proportional to $L_{x}^{2}$; but for $k L_{x}=1$ to 3 , the potential is crudely proportional to $L_{x}^{2}$ divided by 1 to 3 . If we assume that $k \lambda_{D}=0.3$ for the ion acoustic waves (Mangeney et al., 1999), $k L_{x}$ varies between 0.5 and 3 because the Debye length $\lambda_{D}$ is comprised between 5 and $30 \mathrm{~m}$ in our solar wind sample. To take crudely into account the bias of the antenna response, $E^{2}$ will be multiplied by $15 / \lambda_{D}$ when $\lambda_{D} \leq 15 \mathrm{~m}$, i.e. when $\lambda \leq 300 \mathrm{~m}$. An analysis of the TNR spectra allows for the subtraction of the thermal fluctuations, and thus, for the first time, a continuous measure of the level of the non-thermal spectra that are the spectral counterparts of the TDS waveforms.

In Sect. 3, we recall the proportion of coherent electrostatic waves that are observed to be weak double layers (Mangeney et al., 1999), and give the proportion of CEW observed above $4 \mathrm{kHz}$. We then determine the typical spectrum of a CEW, which is the average over the spectra of the TDS waveforms observed during a total time interval $T=38$ days. With the TNR instrument operating continuously, we determine the average of the non-thermal spectral energy over the same interval $T$ (Sect. 4), above $4 \mathrm{kHz}$. The number of CEW above $4 \mathrm{kHz}$ during $T$ will be given by the ratio of the TNR average non-thermal spectrum over the typical spectrum of a TDS event (Sect. 5). We finally obtain the average rate of occurrence of WDL in the whole ion acoustic frequency range, in the solar wind at $1 \mathrm{AU}$.

During the studied period ( 38 days, from 20 May to 26 June 1995), the bit rate on TDS is high every two days. Thus, the occurrence of the weak double layers will be determined with 19 days of data. This interval is relatively short, but it is typical of the solar wind close to the minimum of solar activity because Wind has explored high-speed streams as well as low-speed streams (Mangeney et al., 1999; Lacombe et al., 2000).

It is well known that, in the foreshock of the Earth's bow shock, electrostatic waves can be generated by backstreaming electrons or protons (Filbert and Kellogg, 1979). We do not want to consider these waves, but rather only the waves generated in the free solar wind. For an analysis (Sect. 6) of the correlations between the level of the non-thermal ion acoustic waves and the properties of the solar wind, we have thus withdrawn the hourly intervals during which Wind is in the foreshock, i.e. downstream of a field line tangent to the bow shock, or during which Wind is upstream of this field line but at less than $50 R_{E}$ (along the GSE- $X$ axis) of the foreshock boundary. Only $5 \%$ of the hourly intervals have been withdrawn, corresponding to a geometry in which the interplanetary magnetic field is nearly radial, because Wind was close to the Lagrange point, at more than $200 R_{E}$ from the Earth.

In this study, we use hourly averages of the Key Parameters data: the magnetic field components (MFI experiment, 

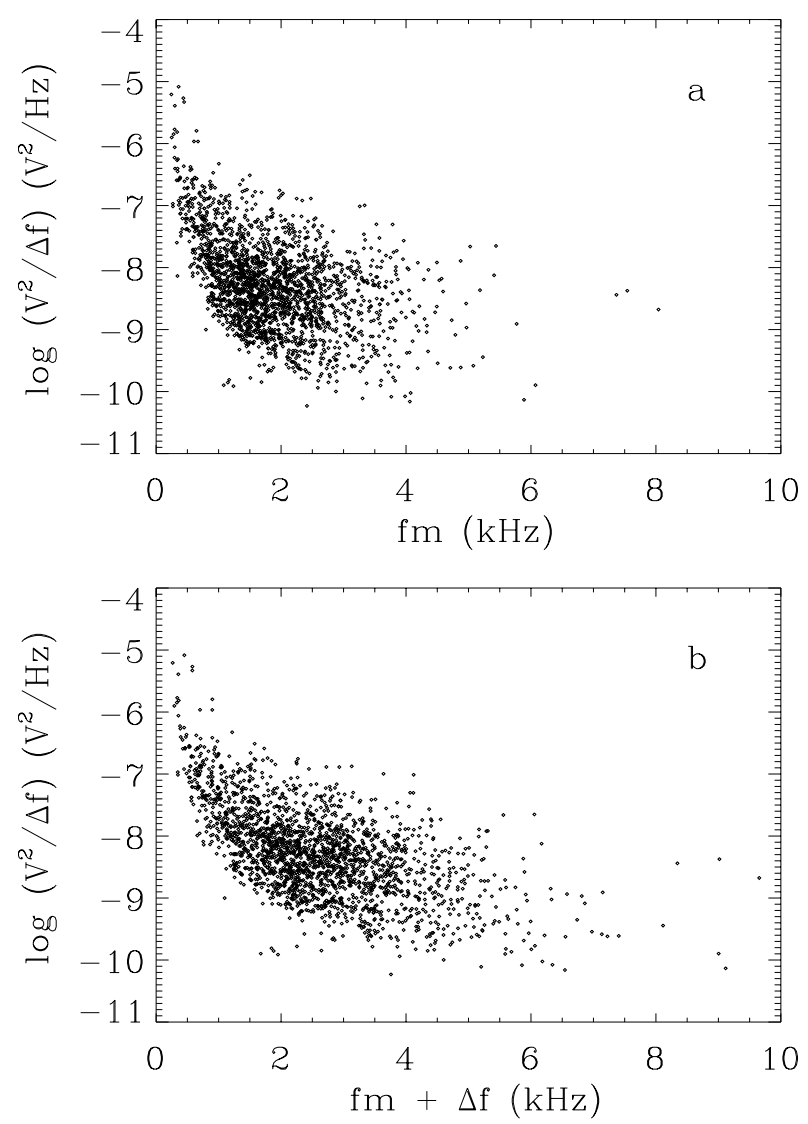

Fig. 2. Spectral power of the electric potential in the coherent electrostatic waves. (a) as a function of their central frequency $f_{m}$. (b) as a function of their maximum frequency $f_{m}+\Delta f$.

Lepping et al., 1995), the electron and proton temperatures (3D-Plasma experiment, R. Lin et al., 1995), the proton density and the solar wind velocity (SWE experiment, Ogilvie et al., 1995). The detailed electron distribution functions (R. Lin et al., 1995) have been integrated to give hourly averages of the components of the heat flux vector, and of the parallel to perpendicular temperature ratio $T_{e \|} / T_{e \perp}$ (Salem et al., 2001).

\section{Properties of the coherent electrostatic waves (CEW)}

To analyse the sample of 2160 CEW detected by TDS during 19 days, we have calculated the central frequency $f_{m}$ of each waveform, its bandwidth $\Delta f$ and its mean square electric field $E^{2}$ in $\mathrm{V}^{2} / \mathrm{m}^{2}$ (Mangeney et al., 1999). The CEW display two main typical shapes (Fig. 1):

- sinusoidal wave packets (Fig. 1a) lasting more than $5 \mathrm{ms,}$ with a relative bandwidth $\Delta f / f_{m}<0.4$ (Fig. 1b); 985

(about $46 \%$ ) of the CEW are wave packets.
- non-sinusoidal isolated structures (Fig. 1c) lasting about $1 \mathrm{~ms}$ : these electrostatic spikes have a larger relative bandwidth (Fig. 1d). Assuming that these isolated structures are one-dimensional, Mangeney et al. (1999) found a potential difference $\Delta \Phi$ across the structure

$\Delta \Phi \geq 10^{-3} V, \quad e \Delta \Phi / k_{B} T_{e} \simeq 10^{-4}-10^{-3}$,

where $k_{B}$ is the Boltzman constant and $e$ the electron charge. Such potential differences imply that the corresponding structures are WDL: 626 (about 29\%) of the CEW are weak double layers.

Figure 2 displays the mean square electric potential per Hertz of each event $V^{2} / \Delta f=E^{2} L_{x}^{2} / \Delta f$ as a function of $f_{m}(\mathrm{kHz})$, and as a function of $f_{m}+\Delta f$, which is a measure of the highest frequency reached by the event. The sharp lower bound of the electric potential in Fig. 2 is produced by the threshold of the TDS receiver, which increases when the frequency decreases. We note (Fig. 2a) that $f_{m}$ varies between 0.2 and $8 \mathrm{kHz} ; f_{m}+\Delta f$ (Fig. 2b) is larger than $4 \mathrm{kHz}$ in $11 \%$ of the CEW: the TNR receiver that operates above $4 \mathrm{kHz}$ will be able to detect $11 \%$ of the CEW in the solar wind.

The upper line of Fig. 5a (Sect. 5) gives the spectral density $\mathrm{V}^{2}(f)$ of the CEW averaged over the 2160 waveform spectra. The largest part of the spectral energy is below $4 \mathrm{kHz}$; but between 4 and $6 \mathrm{kHz}$ there is a non-negligible energy that can be compared with the TNR observations (next Section). This comparison will allow for the determination of the rate of occurrence of weak double layers.

\section{Properties of the electrostatic fluctuations on TNR}

\subsection{Comparison with the TDS data}

Figure 3 gives the temporal profile of the electrostatic fluctuations (in $\mathrm{V}^{2} / \mathrm{Hz}$ ) measured on the Thermal Noise Receiver (TNR) at three frequencies between 4 and $8 \mathrm{kHz}$, on 21 May 1995. During this six hour interval, the plasma frequency is nearly constant, $f_{p e} \simeq 23-25 \mathrm{kHz}$. Around 10:00 UT, the only fluctuations are the thermal fluctuations, the level of which decreases slightly when the frequency increases. From 05:00 to 08:00 UT, very sporadic non-thermal fluctuations are observed. Their intensity, and thus their rate of occurrence, decreases when the frequency increases. Are these TNR fluctuations the spectral counterparts of the waveforms seen by TDS?

Histograms of the intensity of the TNR electrostatic fluctuations, observed on the same day at two frequencies, are shown in Fig. 4. The dashed lines correspond to an interval of thermal noise, 09:00 to 10:00 UT (see Fig. 3). The solid lines correspond to a longer interval (00:00 to 14:00 UT) during which the density $N_{e}$, the electron and proton temperatures $T_{e}$ and $T_{p}$, and the wind speed $V_{s w}$ remained nearly constant. The solid line histograms are made of two components: 

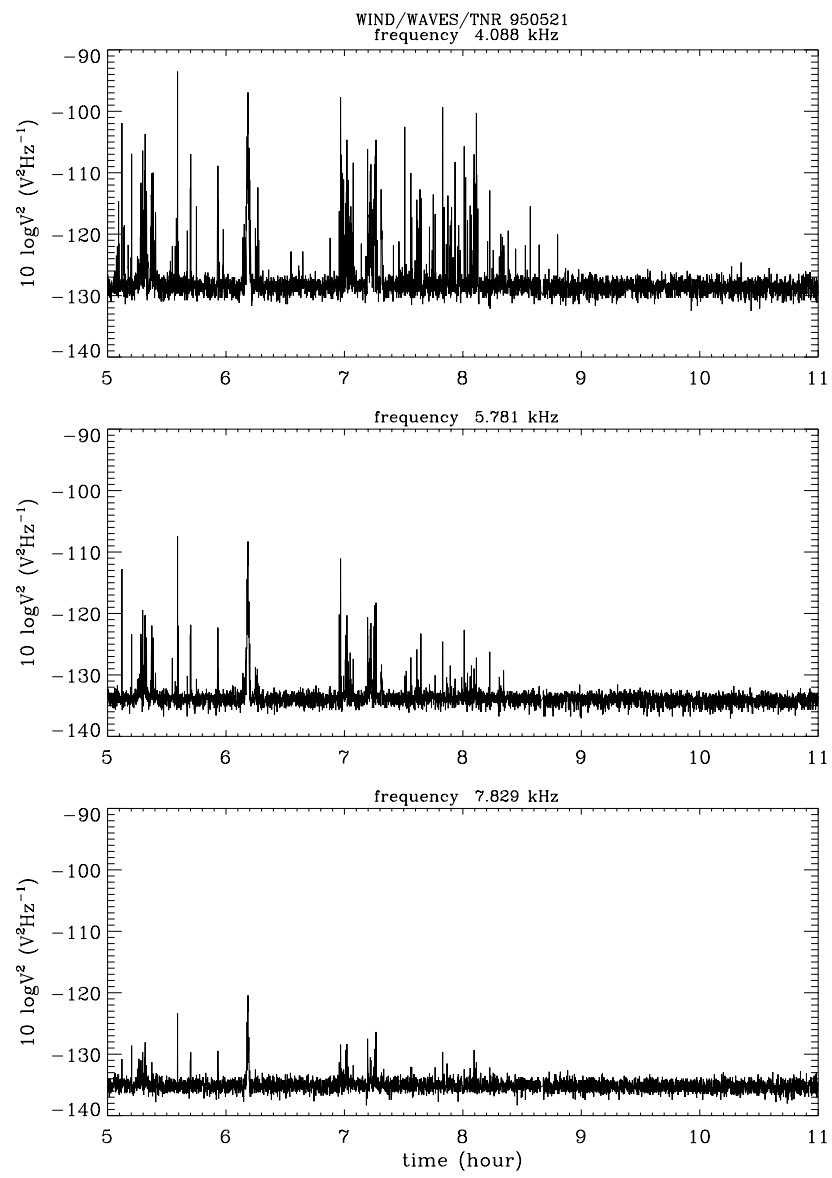

Fig. 3. Time profile of the spectral power of the electric potential at three frequencies on TNR, during six hours (sampling time: $4.5 \mathrm{~s}$ ).

- a low intensity component (peaks at $10^{-13}$ and $310^{-14} \mathrm{~V}^{2} / \mathrm{Hz}$, respectively, at 4.27 and $7.18 \mathrm{kHz}$ ). Its intensity distribution is Gaussian, and corresponds to the thermal ion acoustic noise which depends on $N_{e}$, $V_{s w}, T_{e}$ and $T_{p}$ (Issautier et al., 1999);

- a high intensity component which is called non-thermal: it is not observed between 09:00 and 10:00 UT (dashed line histogram). Its intensity distribution is more or less a power law.

Around $4 \mathrm{kHz}$, the spectral power on TNR is generally below $10^{-11} \mathrm{~V}^{2} / \mathrm{Hz}$ (Figs. 3 and 4 ), while the average spectrum on TDS reaches $10^{-10} \mathrm{~V}^{2} / \mathrm{Hz}$ (Fig. 5a). This discrepancy is simply due to the fact that the spectra of the TDS events are made with a signal integrated during $17.07 \mathrm{~ms}$, while the integration time for the TNR spectra is $1.472 \mathrm{~s}, 86$ times larger: if there is only one isolated event like the WDL of Fig. 1c during $1.472 \mathrm{~s}$, then the corresponding TNR spectrum will be 86 times less intense than the TDS spectrum. We see on Fig. 3 that a large part of the spikes observed at $4.088 \mathrm{kHz}$ are not observed at $5.781 \mathrm{~Hz}$. They probably correspond to narrow band waveforms, as in Figs. 1a and 1b. Broad band waveforms (Figs. 1c and 1d) can be observed above $6 \mathrm{kHz}$.
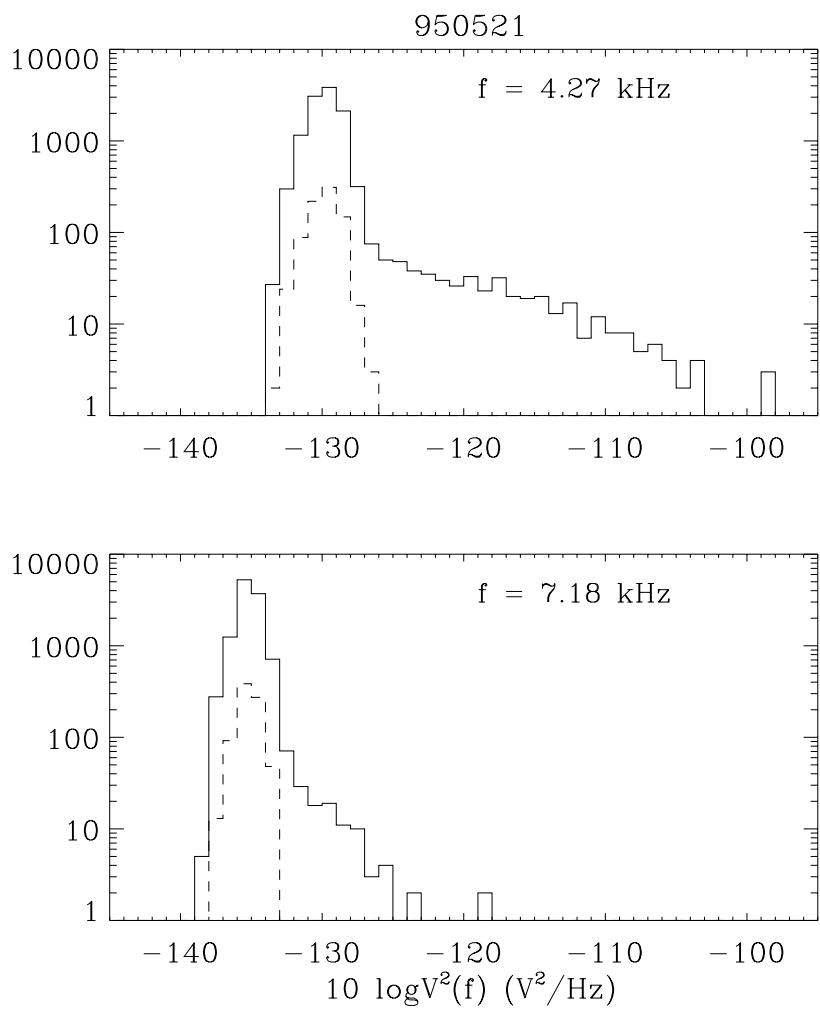

Fig. 4. Solid lines: histograms of the TNR spectral power at two frequencies, during $14 \mathrm{~h}$. Dashed lines: histograms at the same frequency during one hour with purely thermal fluctuations.

Thus, the non-thermal fluctuations of the TNR receiver are the spectral counterparts of the TDS waveforms: if we take into account the ratio between the integration times on TNR and TDS, then the fluctuations have the same intensity in their common frequency range, 4 to 6 or $8 \mathrm{kHz}$.

\subsection{Percentage of non-thermal fluctuations on TNR}

Histograms similar to those of Fig. 4 have been drawn every hour during 38 days, in the 32 frequencies of TNR between 4 and $16 \mathrm{kHz}$. They allow for the determination of the hourly critical value $\mathrm{V}_{c}^{2}(f)$, in $\mathrm{V}^{2} / \mathrm{Hz}$, below which the histogram is Gaussian. A TNR spectrum will be considered as nonthermal at a frequency $f$ if its intensity $\mathrm{V}^{2}(f)$ is larger than $\mathrm{V}_{c}^{2}(f)$. The percentage $P(f)$ of non-thermal spectra is then

$P(f)=100 N_{N T}(f) / N_{\text {tot }}$,

where $N_{\text {tot }}$ is the total number (about 800 ) of spectra observed during one hour, and $N_{N T}(f)$ is the number of spectra which are non-thermal at the frequency $f$ during the same hour. Figure $5 \mathrm{~b}$ shows that the average of $P(f)$ over 38 days increases when $f$ decreases. This increase is weak, so that the average of $P(f)$ would have not reached $10 \%$ if TNR had operated at 1 or $2 \mathrm{kHz}$, where the CEW are more frequent (Fig. 2a). 

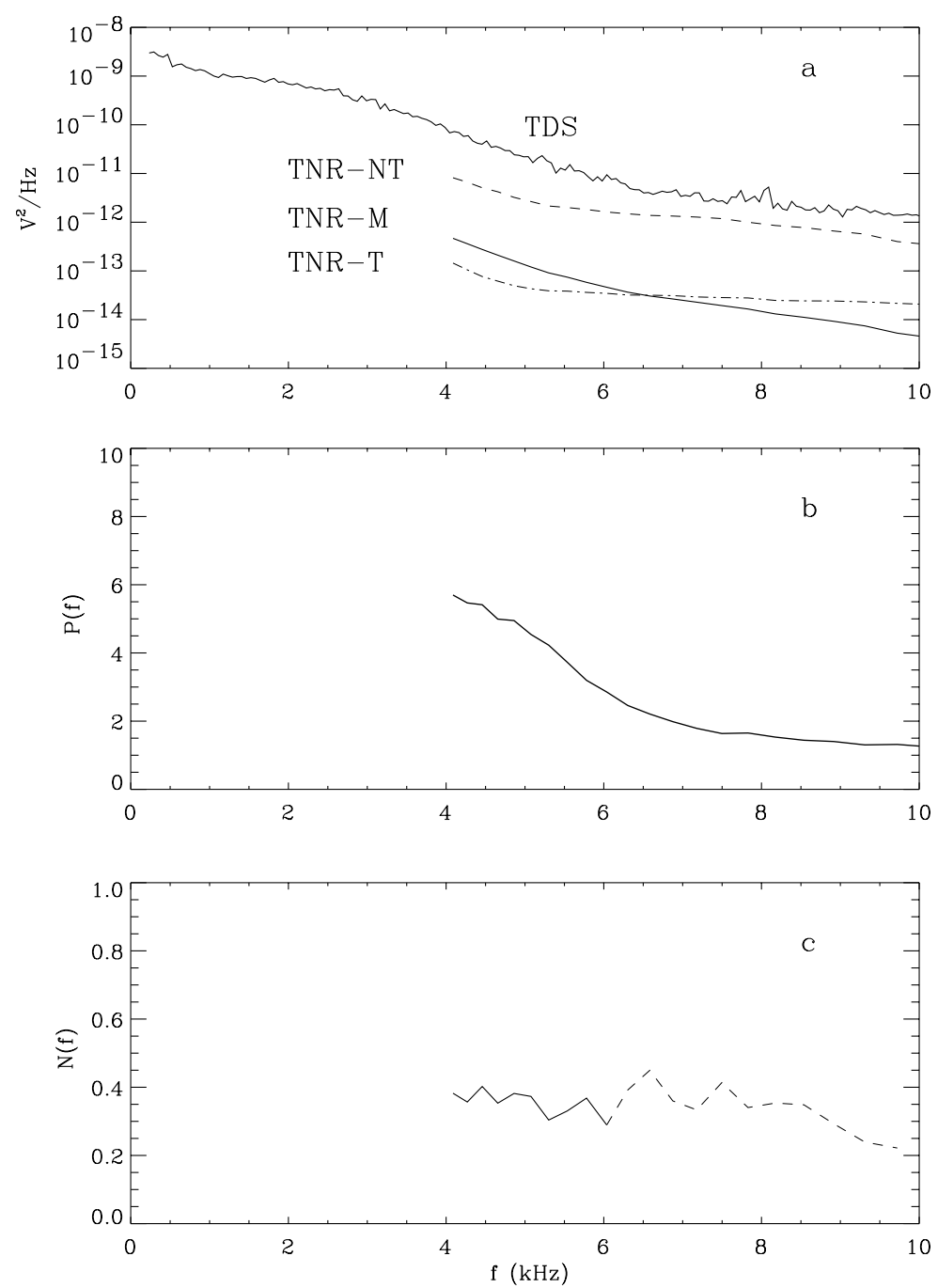

Fig. 5. (a) The upper line is the average spectrum of the 2160 coherent electrostatic waves detected by TDS from 20 May to 26 June 1995. NT is the nonthermal TNR spectrum averaged over the sample of non-thermal spectra; $T$ is the thermal spectrum averaged over the sample of thermal spectra. The solid line $M$ is the total intensity of the nonthermal TNR spectra divided by the total number of spectra, thermal and nonthermal; it is thus a time average of the non-thermal spectral energy. (b) Average over 38 days of the percentage of non-thermal spectra (Eq. 2), as a function of the frequency. (c) the rate of occurrence as a function of the frequency, in $s^{-1}$ (Eq. 3), of coherent electrostatic waves in the solar wind at $1 \mathrm{AU}$.

\subsection{Average TNR spectra}

On Fig. 5a, the dashed line $T N R-T$ is the spectrum of the purely thermal fluctuations averaged over the sample of thermal spectra, and the dashed line $T N R-N T$ the spectrum of the non-thermal fluctuations averaged over the sample of non-thermal spectra during 38 days. The solid line spectrum labelled $M$ is the average of the non-thermal TNR spectral energy over the total number of spectra, thermal plus nonthermal; it is thus a time average of the non-thermal energy over 38 days. (The increase of the slope of the average thermal spectrum $T$ below $5 \mathrm{kHz}$ is an artifact due to the background noise: the actual background noise was sometimes larger than the background noise subtracted from the raw data (Salem, 2000), so that the measured fluctuations were sometimes overestimated between 4 and $6 \mathrm{kHz}$. This uncertainty on the background noise does not spoil the $M$ and $N T$ spectra which are calculated with the non-thermal fluctuations, well above the background).

\section{Occurrence of the coherent electrostatic waves (CEW)}

The average number of CEW observed per second in the solar wind will be given by a comparison of the average spectrum $\mathrm{V}_{T D S}^{2}$ of the TDS waveforms (upper line in Fig. 5a) with the time-averaged spectrum $\mathrm{V}_{T N R}^{2}$ of the TNR nonthermal fluctuations (labelled $M$ in Fig. 5a). As noted in Sect. 4.1, the ratio between the integration times on TNR and TDS is $b=86$. If there is only one TDS event during the TNR integration time $\tau=1.472 \mathrm{~s}$, then its TNR intensity at a given frequency will be $\mathrm{V}_{T N R}^{2}=\mathrm{V}_{T D S}^{2} / b$. The ratio

$N(f)=b V_{T N R}^{2}(f) /\left(\tau V_{T D S}^{2}(f)\right)$

thus gives the number of CEW observed in the solar wind during $1 \mathrm{~s}$, above $4 \mathrm{kHz} ; N(f)$ is drawn in Fig. $5 \mathrm{c}$. We do not consider $N(f)$ above $6 \mathrm{kHz}$ (dashed line) because the number of CEW is very low (Fig. 2). Between 4 and $6 \mathrm{kHz}$ (solid line), the ratio $N(f)$ is nearly constant, and its average value is 0.36: thus, during one second, there is $0.36 \mathrm{CEW}$ above $4 \mathrm{kHz}$. We have seen (Sect. 3) that $11 \%$ of the CEW 

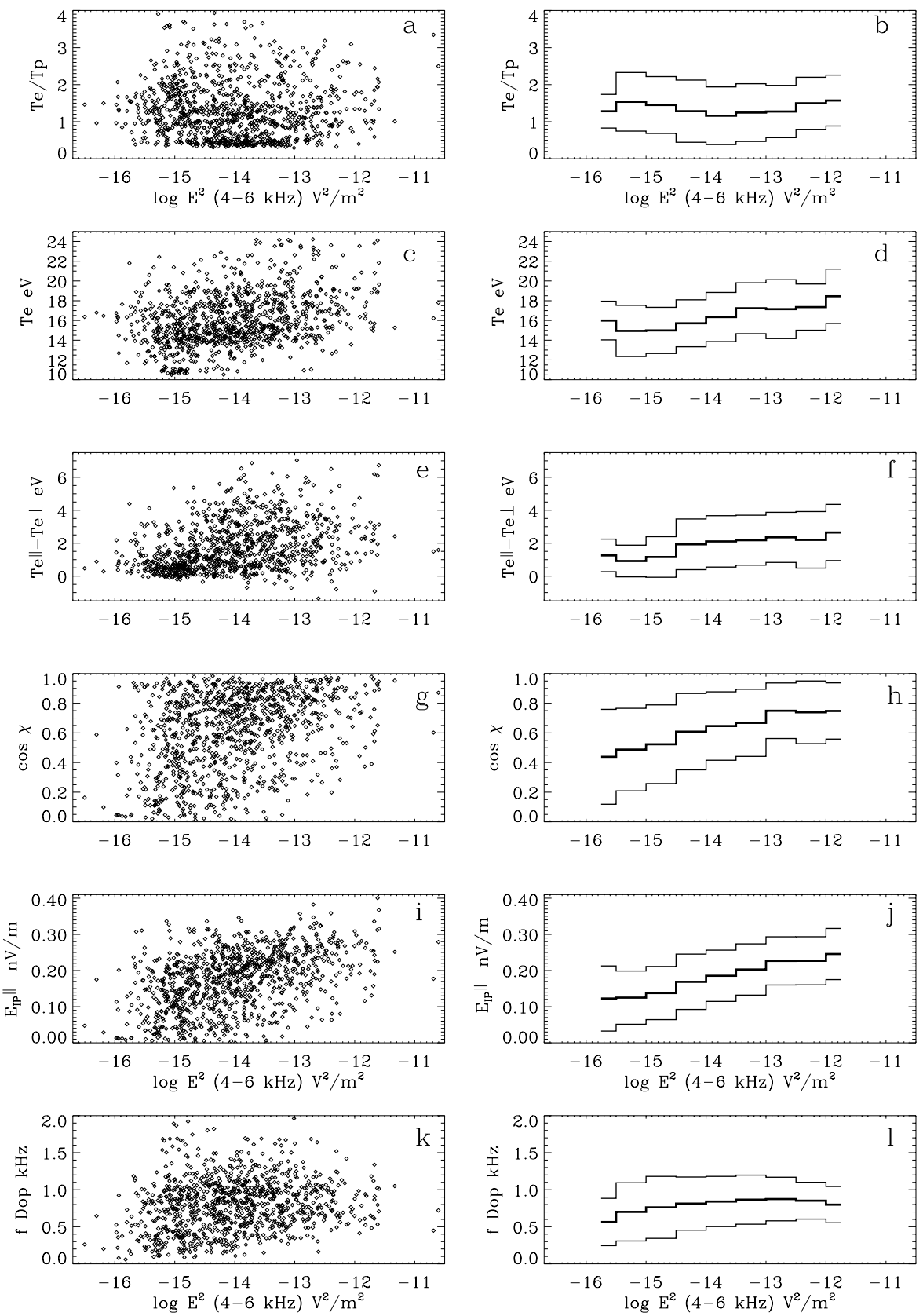

Fig. 6. In the free solar wind (not connected to the Earth's bow shock), hourly averages of solar wind parameters as functions of the hourly energy $E^{2}$ in non-thermal ion acoustic waves between 4 and $6 \mathrm{kHz}$. Left-hand panels: scatter plots. Right panels: average and standard deviation in equal bins of $\log E^{2}$. Ordinates: (a) and (b) the temperature ratio $T_{e} / T_{p} ;$ (c) and (d) the electron temperature; (e) and (f) the electron temperature anisotropy; $(\mathbf{g})$ and (h) the cosine of the acute angle between the magnetic field and the radial direction; (i) and (j) the interplanetary electric field parallel to the spiral magnetic field in a two-fluid model (Eqs. 10 to 13); (k) and (l) the estimated Doppler shift of ion acoustic waves in the solar wind (Eq. 14). For $E^{2}$, we have crudely corrected a bias due to the dipole antenna response for small wavelengths (see Sect. 2). are observed above $4 \mathrm{kHz}$. Thus, the number of CEW per second from 0 to $6 \mathrm{kHz}$ is

$N_{C E W} \simeq 3.3 s^{-1}$.

Since about $29 \%$ of the solar wind CEW are weak double layers (Sect. 3),

$N_{W D L} \simeq 1 s^{-1}$.

During the average travel time of the solar wind from the Sun to the Earth $\left(t \simeq 310^{5} \mathrm{~s}\right)$, the total number of WDL will be $N_{W D L} t \simeq 310^{5}$. The potential jumps measured on Wind (Mangeney et al., 1999) are between $210^{-4} \mathrm{~V}$ and $510^{-2} \mathrm{~V}$, with an average value of $410^{-3} \mathrm{~V}$, for a sample of 77 events arbitrarily selected. As the selection has probably favoured the strongest events, we consider that the average potential jump across a WDL is $\Delta \Phi \simeq 10^{-3} \mathrm{~V}$ to $310^{-3} \mathrm{~V}$.

The total potential jump between the Sun and the Earth is then

$300 \mathrm{~V} \leq \Phi \leq 1000 \mathrm{~V}$.

This estimation relies on the simple assumptions that the rate of occurrence of the WDL and their typical potential jump are constant from the corona to the Earth.

Let us compare $\Phi$ with the exospheric potential $\Phi_{E X} \simeq$ 400 to $500 \mathrm{~V}$, and with the potential $\Phi_{P G}$ at $R=1 \mathrm{AU}$, due to the gradient of the electron pressure in an isotropic plasma 
with a radial magnetic field. Neglecting the electron inertia, we have

$e N_{e} d\left(\Phi_{P G}\right) / d r=d\left(N_{e} k_{B} T_{e}\right) / d r$.

An integration gives

$e \Phi_{P G}=T_{e 0} \frac{2+\alpha}{\alpha}\left[\left(R / r_{0}\right)^{-\alpha}-1\right]$,

where $T_{e 0}$ is the electron temperature in the corona at a distance $r_{0}=4 R_{S}$, and $R=214 R_{S} ; R_{S}$ is the solar radius; $\alpha \simeq 0.6$ is the power law index of the electron temperature which varies like $r^{-\alpha}$ with the distance from the Sun; the density varies like $r^{-2}$. We find that $\Phi_{P G} \simeq 400 \mathrm{~V}$ to $600 \mathrm{~V}$ for $T_{e 0} \simeq 100$ to $150 \mathrm{eV}$. As $\Phi_{P G}$ and $\Phi_{E X}$ are of the same order of magnitude, our observations cannot help to distinguish them, inasmuch as the uncertainty on $\Phi$ (Eq. 6) is large. Our estimate is consistent with a two-fluid model of the solar wind, as well as with an exospheric model.

\section{Energy of the ion acoustic waves in different solar wind regions}

Gurnett et al. (1979) have found correlations between the electric field in the ion acoustic waves and some properties of the solar wind plasma: in spite of a large dispersion of the data, the peak electric field over $36 \mathrm{~min}$ of data, at $5.62 \mathrm{kHz}$, tends to increase when $T_{e} / T_{p}$ increases, when the heat flux increases, and when the solar wind speed decreases. We have not considered the peak electric field, but rather the square electric field $E^{2}$ of the non-thermal fluctuations, averaged over the 800 spectra measured during $1 \mathrm{~h}$, between 4 and $6 \mathrm{kHz}$. As explained in Sect. 2, we have withdrawn from our analysis the intervals during which there was a risk, for Wind, to be in the Earth's foreshock. $E^{2}$ varies between $10^{-16}$ and $10^{-11} \mathrm{~V}^{2} / \mathrm{m}^{2}$. The lowest values $10^{-16}$ to $10^{-14} \mathrm{~V}^{2} / \mathrm{m}^{2}$ are below the thermal electrostatic level corresponding to the $T$ spectrum in Fig. 5a; they are found during hours with very few non-thermal spectra.

Figure 6a displays a scatter plot of $T_{e} / T_{p}$ as a function of $\log E^{2}$. The right-hand panel (Fig. 6b) gives the average value and the standard deviation of the same quantity, in equal bins of $\log E^{2}$. The variation of the peak electric field at $5.62 \mathrm{kHz}$ with $T_{e} / T_{p}$ found by Gurnett et al. (1979) is not observed here, neither in the scatter plot nor in the average profile, for the hourly averages of $E^{2}$. Similarly, we do not find any variation of $\log E^{2}$ with the solar wind speed $V_{s w}$, or the heat flux $Q_{e}$. The electron Coulomb collision frequency $\nu_{e}$ is a function of $N_{e}$ and $T_{e}$

$v_{e}=2.55 v_{e \perp}=2.55710^{-6} N_{e} T_{e}^{-3 / 2} \ln \Lambda$,

where $v_{e \perp}$ is the electron-electron collision frequency for the angular diffusion, and 2.55 is a factor which takes into account the electron-proton and electron- $\alpha$ collisions (Phillips and Gosling, 1990). There is a slight tendency for $\log E^{2}$ to be larger when $T_{e}$ is large (Figs. 6c and 6d), and when the density is low (not shown). Thus, $\log E^{2}$ tends to be larger when there are less collisions. Phillips and Gosling (1990) have shown that the solar wind behaves like a marginally collisional plasma in which the electron temperature anisotropy is regulated by collisions and by expansion. Their model disregards the interplanetary electrostatic field. They find that the electron temperature anisotropy, observed on ISEE 3, is larger when $v_{e}$ is weaker and when the magnetic field is more radial. This last property is also observed in our sample: a comparison of Figs. 6e and $6 \mathrm{~g}$ shows that $T_{e \|}-T_{e \perp}$ is larger when the field is more radial $(\cos \chi \simeq 1)$. We thus find that the intensity of the ion acoustic waves is sensitive to the collisional properties of the plasma and to its expansion rate. But the ion acoustic waves cannot be due to the electron temperature anisotropy instability: indeed, the electron firehose instability generates waves around the proton cyclotron frequency, well below the ion acoustic frequency (Hollweg and Völk, 1970); furthermore, the instability condition is not fulfilled in our sample.

The parameters with which $\log E^{2}$ is related, $T_{e}, T_{e \|}-T_{e \perp}$ and $\cos \chi$ (Figs. 6c, 6e and 6g) do not only play a part in the Coulomb collision frequency and the solar wind expansion, but they also play a part in the interplanetary electrostatic potential. According to Pilipp et al. (1990), the interplanetary electrostatic potential $\Phi_{P G}$ due to the electron pressure gradient, in a two-fluid model (Eq. 7), can be written as a function of $T_{e}, T_{e \|}-T_{e \perp}$ and $\cos ^{2} \chi$ for a spiral magnetic field in the ecliptic plane

$$
\begin{gathered}
e N_{e} \frac{d \Phi_{P G}}{d r}=\frac{d\left[p_{e \perp}+\left(p_{e \|}-p_{e \perp}\right) \cos ^{2} \chi\right]}{d r} \\
+(1 / r)\left(2 \cos ^{2} \chi-\sin ^{2} \chi\right)\left(p_{e \|}-p_{e \perp}\right),
\end{gathered}
$$

where $p_{e \|, \perp}=N_{e} k_{B} T_{e \|, \perp}$ is the electron pressure parallel and perpendicular to the $B$ field. Using the relation

$\cos ^{2} \chi=V_{s w}^{2} /\left(V_{s w}^{2}+\Omega^{2} r^{2}\right)$,

where $\Omega$ is the angular frequency of the Sun's rotation, the radial component of the interplanetary electric field at $1 \mathrm{AU}$ is

$$
\begin{gathered}
E_{r}(V / m)=-\frac{d \Phi_{P G}}{d r}=\left[(2+\alpha) T_{e \perp}+\left(T_{e \|}-T_{e \perp}\right)\right. \\
\left.\left[1+(1+\alpha) \cos ^{2} \chi-2 \cos ^{4} \chi\right]\right] / 1.510^{11},
\end{gathered}
$$

where the temperatures are in $\mathrm{eV}$, and the component of this electric field along the magnetic field is

$E_{I P \|}(V / m)=|\cos \chi| E_{r}$.

Figures $6 \mathrm{i}$ and $6 \mathrm{j}$ indicate that there is a relation between $E_{I P \|}$, calculated at $1 \mathrm{AU}$ for $\alpha=0.6$, and the ion acoustic fluctuation level $E^{2}$ between 4 and $6 \mathrm{kHz}$ : the correlation coefficient between $\log E^{2}$ and $E_{I P \|}$ is 0.45 . With a correlation coefficient of 0.45 and a sample of 991 points, the probability that $\log E^{2}$ and $E_{I P \|}$ are uncorrelated is less than $10^{-6}$. We thus consider that this correlation is significant, inasmuch as the correlation of $\log E^{2}$ with $E_{I P \|}$ is better than the individual correlations of $\log E^{2}$ with $T_{e}(0.32)$, with $T_{e \|}-T_{e \perp}$ 
(0.31) or with $\cos ^{2} \chi(0.32)$. This result indicates that the coherent electrostatic waves are related to the large-scale interplanetary electrostatic field parallel to the magnetic field.

\section{Discussion}

The correlation shown in Figs. 6i and $6 \mathrm{j}$ between the calculated value of the parallel interplanetary electrostatic field in a two-fluid model (Eqs. 12 and 13) and the measured level of the non-thermal ion acoustic fluctuations $E^{2}$ is significant, in spite of a large dispersion of the scatter plot. We have yet to check whether or not this correlation is an artifact of the Doppler effect in the solar wind. We have seen (Fig. 2) that most of the ion acoustic fluctuations were observed on TDS between 0.5 and $3 \mathrm{kHz}$, while TNR can only measure them above $4 \mathrm{kHz}$. Thus, an increase in $E^{2}$ between 4 and $6 \mathrm{kHz}$ on TNR can be simply due to an increase in the Doppler shift, which brings above $4 \mathrm{kHz}$ the waves emitted below $4 \mathrm{kHz}$. The considered electrostatic waves propagate along $\boldsymbol{B}$, with wave vectors in the range $0.1<k \lambda_{D}<0.6$ (Mangeney et al., 1999). The Doppler shift is

$f_{D o p}=\frac{k V_{s w} \cos \left(B, V_{s w}\right)}{2 \pi}=\frac{k \lambda_{D}}{2 \pi} \frac{V_{s w} \cos \left(B, V_{s w}\right)}{\lambda_{D}}$,

where $\left(B, V_{s w}\right)$ is the acute angle between $\boldsymbol{B}$ and $\boldsymbol{V}_{s w}$. Figures $6 \mathrm{k}$ and $6 \mathrm{l}$ display $f_{\text {Dop }}$ calculated with the average value $k \lambda_{D}=0.3$, as a function of $\log E^{2}$. For waves with $E^{2}>10^{-13} \mathrm{~V}^{2} / \mathrm{m}^{2}$, the Doppler effect is important ( 0.5 to $1.5 \mathrm{kHz})$. Since the angle $\left(B, V_{s w}\right)$ is near the angle $\chi$, a part of the relations between $\log E^{2}$ and $\cos \chi$ or $E_{I P \|}$ (Figs. $6 \mathrm{~g}$ and 6i) can be due to the Doppler effect. Similarly, a part of the relations between $\log E^{2}$ and $T_{e}$ (Figs. $6 \mathrm{c}$ and $6 \mathrm{~d}$ ), or between $\log E^{2}$ and the density, can be due to the relation between the Doppler shift $f_{D o p}$ and $\lambda_{D}$, for $k \lambda_{D} \simeq$ constant.

To really test whether the intensity of the ion acoustic waves depends on the solar wind properties and is related to the solar wind expansion, it would be necessary to get rid of the Doppler effect, i.e. to measure the intensity of the ion acoustic waves in their whole frequency domain $(0.2 \mathrm{kHz}$ to 6 or $8 \mathrm{kHz}$ ) and not in a narrow frequency domain around $5 \mathrm{kHz}$. This cannot be done on Wind/TNR which only operates above $4 \mathrm{kHz}$. On Ulysses (see Hess et al., 1998), the lowest frequency is $1.25 \mathrm{kHz}$, with linear frequency steps of $0.75 \mathrm{kHz}$. Unfortunately, there is on Ulysses a frequency sweeping which lasts $128 \mathrm{~s}$, with 2 or 4 measurements during $2 \mathrm{~s}$, at each frequency. With this low time and frequency resolution, the distinction between the thermal and the nonthermal spectra, using histograms similar to Fig. 4, would be less clear.

\section{Conclusion}

The Time Domain Sampler (TDS) instrument on Wind has allowed for the detection of coherent electrostatic waveforms in the solar wind. The central frequency of these waveforms is in the ion acoustic range 0.2 to $8 \mathrm{kHz}$. Some of these waveforms are weak double layers (WDL): the electrostatic potential drops over a few Debye lengths, and these drops are directed towards the Earth, in the same sense as the largescale interplanetary electrostatic potential between the Sun and the Earth (Mangeney et al., 1999). The rate of occurrence of the WDL cannot be determined with the TDS instrument alone, which does not operate continuously. The Thermal Noise Receiver (TNR) on Wind operates continuously in the ion acoustic spectral domain, and measures the electrostatic fluctuations above $4 \mathrm{kHz}$. A comparison of the time average of the TNR non-thermal spectral energy and of the average TDS spectrum, over a period of 38 days, has allowed for the determination of the rate of occurrence of WDL in the solar wind, $N_{W D L} \simeq 1 \mathrm{~s}^{-1}$. Assuming that this rate of occurrence observed at $1 \mathrm{AU}$ is constant during the travel time of the solar wind, the total potential drop between the Sun and the Earth is $300 \mathrm{~V} \leq \Phi \leq 1000 \mathrm{~V}$, which compares well with the potentials implied by the two-fluid solar wind model and the exospheric model. A first experimental evidence of the existence of the large-scale interplanetary electric potential is thus given by the determination of the rate of occurrence of the weak double layers, the scale of which is a few Debye lengths.

We have subtracted the thermal fluctuations from the TNR spectra and determined the hourly averages $E^{2}\left(\mathrm{~V}^{2} / \mathrm{m}^{2}\right)$ of the non-thermal ion acoustic spectral energy integrated between 4 and $6 \mathrm{kHz} . E^{2}$ does not depend on the wind speed, nor on the ratio $T_{e} / T_{p}$, nor on the electron heat flux. But we find a correlation between $E^{2}$ and the interplanetary electric field parallel to the spiral magnetic field, calculated with the model of Pilipp et al. (1990). This correlation could be considered as a second evidence of the relation between waves in the ion acoustic frequency range and the large-scale interplanetary potential. We show, however, in the Discussion that this second evidence is weakened by the fact that the TNR receiver only operates above $4 \mathrm{kHz}$, while most of the ion acoustic waves, in spite of a strong Doppler shift, are observed on TDS around $1-2 \mathrm{kHz}$.

Acknowledgements. The WAVES experiment on WIND was built by teams of the University of Minnesota, the University of Iowa and the Observatoire de Paris, Meudon, with support of NASA/GSFC. The french contribution is supported by the Centre National d'Etudes Spatiales and the Centre National de la Recherche Scientifique. We are very grateful to the teams of the Three Dimensional Plasma experiment (PI R. P. Lin), of the Magnetic Field Investigation experiment (PI R. P. Lepping) and of the Solar Wind experiment (PI K. W. Ogilvie).

Topical Editor E. Antonucci thanks G. Mann and another referee for their help in evaluating this paper.

\section{References}

Bougeret, J.-L., Kaiser, M. L., Kellogg, P. J., Manning, R., Goetz, K., Monson, S. J., Monge, N., Friel, L., Meetre, C. A., C. Perche, C., Sitruk, L., and Hoang, S.: WAVES: the radio and plasma 
wave investigation on the WIND spacecraft, Space Sci. Rev., 71, 231-263, 1995.

Filbert, P. C. and Kellogg, P. J.: Electrostatic noise at the plasma frequency beyond the Earth's bow shock, J. Geophys. Res., 84, 1369-1381, 1979.

Gary, S. P., Skoug, R. M., and Daughton, W.: Electron heat flux constraints in the solar wind, Physics of Plasmas, 6, 2607-2612, 1999.

Gurnett, D. A.: Waves and instabilities, in: Physics of the inner heliosphere II, (Eds) Schwenn, R. and Marsch, E., Springer-Verlag, 135-157, 1991.

Gurnett, D. A., Marsch, E., Pilipp, W., Schwenn, R., and Rosenbauer, H.: Ion acoustic waves and related plasma observations in the solar wind, J. Geophys. Res., 84, 2029-2038, 1979.

Hess, R. A., MacDowall, R. J., Goldstein, B., Neugebauer, M., and Forsyth, R. J.: Ion acoustic-like waves observed by Ulysses near interplanetary shock waves in the three-dimensional heliosphere, J. Geophys. Res., 103, 6531-6541, 1998.

Hollweg, J. V. and Völk, H. J.: New plasma instabilities in the solar wind, J. Geophys. Res., 75, 5297-5309, 1970.

Issautier, K., Meyer-Vernet, N., Moncuquet, M., Hoang, S., and McComas, D. J.: Quasi-thermal noise in a drifting plasma: theory and application to solar wind diagnostic on Ulysses, J. Geophys. Res., 104, A, 6691-6704, 1999.

Kellogg, P. J.: Fluctuations and ion isotropy in the solar wind, Astrophys. J., 528, 480-485, 2000.

Lacombe, C., Salem, C., Mangeney, A., Steinberg, J.-L., Maksimovic, M., and Bosqued, J.-M.: Latitudinal distribution of the solar wind properties in the low- and high-pressure regimes: Wind observations, Ann. Geophysicae, 18, 852-865, 2000.

Lemaire, J. and Scherer, M.: Kinetic models of the solar wind, J. Geophys. Res., 76, 7479-7490, 1971.

Lepping, R. P., Acũna, M. H., Burlaga, L. F., Farrell, W. M., Slavin, J. A., Schatten, K. H., Mariani, F., Ness, N. F., Neubauer, F. M., Whang, Y. C., Byrnes, J. B., Kennon, R. S., Panetta, P. V., Scheifele, J., and Worley, E. M.: The WIND magnetic field investigation, Space Sci. Rev., 71, 207-229, 1995

Lin, N., Kellogg, P. J., MacDowall, R. J., Scime, E. E., Balogh, A., Forsyth, R. J., McComas, D. J., and Phillips, J. L.: Very low frequency waves in the heliosphere: Ulysses observations, J. Geophys. Res., 103, 12 023-12 035, 1998.

Lin, N., Kellogg, P. J., MacDowall, R. J., and Gary, S. P.: Ion acoustic waves in the heliosphere, Space Sci. Rev., 97, 193-196, 2001.

Lin, R. P., Anderson, K. A., Ashford, S., Carlson, C., Curtis, D.,
Ergun, R., Larson, D., McFadden, J., McCarthy, M., Parks, G. K., Rème, H., Bosqued, J.-M., Coutelier, J., Cotin, F., d'Uston, C., Wenzel, K.-P., Sanderson, T. R., Henrion, J., Ronnet, J. C., and Paschmann, G.: A three-dimensional plasma and energetic particle investigation for the WIND spacecraft, Space Sci. Rev., 71, 125-153, 1995

Mangeney, A., Salem, C., Lacombe, C., Bougeret, J.-L., Perche, C., Manning, R., Kellogg, P. J., Goetz, K., Monson, S. J., and Bosqued, J.-M.: WIND observations of coherent electrostatic waves in the solar wind, Ann. Geophysicae, 17, 307-320, 1999.

Ogilvie, K. W., Chornay, D. J., Fitzenreiter, R. J., Hunsaker, F., Keller, J., Lobell, J., Miller, G., Scudder, J. D., Sittler, E. C., Torbert, R. B., Bodet, D., Needell, G., Lazarus, A. J., Steinberg, J. T., Tappan, J. H., Mavretic, A., and Gergin, E.: SWE, a comprehensive plasma instrument for the WIND spacecraft, Space Sci. Rev., 71, 55-77, 1995.

Phillips, J. L. and Gosling, J. T.: Radial evolution of solar wind thermal electron distributions due to expansion and collisions, $\mathrm{J}$ Geophys. Res., 95, 4217-4228, 1990.

Pierrard, V., Issautier, K., Meyer-Vernet, N., and Lemaire, J.: Collisionless model of the solar wind in a spiral magnetic field, Geophys. Res. Lett., 28, 223-226, 2001.

Pilipp, W. G., Miggenrieder, H., Mühlhäuser, K.-H., Rosenbauer, R., and Schwenn, R.: Large-scale variations of thermal electron parameters in the solar wind between 0.3 and $1 \mathrm{AU}, \mathrm{J}$. Geophys. Res., 95, 6305-6329, 1990.

Salem, C.: Ondes, turbulence et phénomènes dissipatifs dans le vent solaire à partir des observations de la sonde WIND, Thèse, Université de Paris 7, 2000.

Salem, C., Mangeney, A., and Bougeret, J.-L.: Coherent electrostatic nonlinear waves in collisionless space plasmas, in: Lecture notes in Physics, vol. 536, Nonlinear MHD waves and turbulence, Proceedings of the workshop held in Nice (France), 14, Decembre 1998, (Eds) Passot, T. and Sulem, P. L., SpringerVerlag, 251-268, 1999.

Salem, C., Bosqued, J.-M., Larson, D. E., Mangeney, A., Maksimovic, M., Perche, C., Lin, R. P., and Bougeret, J.-L.: Determination of accurate solar wind electron parameters using particle detectors and radio wave receivers, J. Geophys. Res., 106, 21 701-21 717, 2001

Scudder, J. D., Mangeney, A., Lacombe, C., Harvey, C. C., Wu, C. S., and Anderson, R. R.: The resolved layer of a collisionless, high $\beta$, supercritical, quasi-perpendicular shock wave, 3. Vlasov electrodynamics, J. Geophys. Res., 91, 11 075-11 097, 1986. 